\title{
Ascitis pancreática traumática, manejo conservador: Reporte de un caso
}

\author{
Silvia Inés Guerrero M. ${ }^{1}$, Ada Mercedes Bustos G. ${ }^{1}$, \\ Sergio Andrés Castañeda ${ }^{1}$ y Mayra Zulay Jaimes S. ${ }^{2}$
}

'Departamento de Cirugía de la Universidad Industrial de Santander.

${ }^{2}$ Universidad Industrial de Santander. Bucaramanga, Santander, Colombia.

Recibido 2020-09-20 y aceptado 2020-11-23

Correspondencia a: Dra. Silvia Inés Guerrero M dra.silvia.guerrero@hotmail.

\section{Post-traumatic pancreatic ascites, conservative management: A case}

Aim: To present the case of a young adult male patient, with pancreatic ascites secondary to pancreatic duct injury due to blunt abdominal trauma, treated successfully with conservative therapy. Materials and Method: Data and images were obtained from the clinical chart of the "Hospital Universitario de Santander" with prior informed consent. Results: 21 -year-old male patient with a blunt abdominal trauma background, who consulted for distension, progressive abdominal pain, and subjective weight loss. Due to suspicion of a pancreatic duct injury, a magnetic resonance cholangiopancreatography was requested, which showed an alteration of the proximal segment of the main pancreatic duct associated with a cystic lesion at the anterior border of the junction between the pancreatic head and body. Treatment consisted of a 4-week conservative therapy with the placement of an abdominal drain, bowel rest, associated with total parenteral nutrition and somatostatin analogs. Discussion: A 5\% of blunt abdominal trauma can cause pancreatitis and pancreatic duct leaks. Conclusion: Pancreatic duct injury should be suspected in every patient with blunt abdominal trauma. Management must be done with careful and thorough planning.

Key words: trauma; abdomen; pancreatic duct; fluid; ascites.

\section{Resumen}

Objetivo: Presentar el caso de un paciente masculino adulto joven, con ascitis pancreática secundaria a lesión del conducto pancreático por trauma abdominal cerrado, tratado con éxito con terapia conservadora. Materiales y Método: Datos e imágenes recopilados de la historia clínica del Hospital Universitario de Santander, previo consentimiento informado. Resultados: Sexo masculino de 21 años con antecedente de trauma abdominal cerrado, quien consulta por distensión, dolor abdominal progresivo y pérdida de peso no cuantificada. Ante sospecha de lesión de conducto pancreático se solicitó pancreatografía por resonancia magnética que evidencia una alteración del segmento proximal del conducto pancreático principal asociado a lesión quística en el borde anterior de la unión de la cabeza con el cuerpo pancreático. Se decidió manejo conservador por 4 semanas con colocación de dren abdominal, reposo intestinal, asociado a nutrición parenteral total y análogos de somatostatina. Discusión: Un 5\% del trauma abdominal cerrado puede provocar pancreatitis y fugas en el conducto pancreático. Conclusión: La lesión del conducto pancreático principal debe sospecharse en todos los pacientes con trauma abdominal cerrado. El manejo debe realizarse con una planificación cuidadosa y exhaustiva.

Palabras clave: trauma; abdomen; conducto pancreático; ascitis.

\section{Introducción}

La lesión pancreática secundaria a trauma abdominal cerrado puede manifestarse según el grado de compromiso del parénquima y del conducto pancreático principal ${ }^{1}$, teniendo una presentación clínica inespecífica, necesitando una alta sospecha diagnóstica según el mecanismo del trauma². El trauma pancreático es poco común, puesto que la ubicación retroperitoneal del órgano ofrece una protección relativa; sin embargo, se presenta en aproximadamente el $5 \%$ de los pacientes con traumatismo abdominal cerrado, con una mortalidad de hasta del $30 \%{ }^{3}$, siendo el estado del conducto pancreático el determinante más importante junto con un diagnóstico tardío, aumentando la mortalidad hasta un $17 \%{ }^{2}$. 
Gougeon y cols. ${ }^{4}$, informaron por primera vez en 1976, el uso de colangiopancreatografía retrógrada endoscópica (CPRE) en el diagnóstico de lesión pancreática; actualmente la CPRE temprana, es uno de los métodos más útiles para identificar la lesión del conducto pancreático principal ${ }^{2}$, proporcionando tanto una confirmación diagnóstica, como también la posibilidad de un manejo temprano eficaz. La dificultad en el diagnóstico clínico y paraclínico temprano hace necesario el uso de diferentes estudios de imagen como el ultrasonido abdominal, la tomografía computarizada con protocolo pancreático (sensibilidad del 91\% y especificidad del $91 \%{ }^{5}$ ), la colangiopancreatografía por resonancia magnética (sensibilidad del 95\% a 99\% $\%^{6}$ ) y ultrasonido endoscópico ${ }^{1,7}$.

El manejo de lesiones del conducto pancreático no está estandarizado, sin embargo, existen herramientas conservadoras, quirúrgicas, radiológicas y endoscópicas, que pueden ser usadas de forma aislada o en conjunto. El manejo conservador incluye reposo intestinal, nutrición parenteral total, administración de análogos de somatostatina y reemplazo de enzimas pancreáticas. Las intervenciones endoscópicas incluyen drenaje interno de colecciones pancreáticas (cistogastrostomía, cistoduodenostomía) y colocación de stents transpapilares, la terapia radiológica se basa en drenaje percutáneo externo de las colecciones y, por último, en el manejo quirúrgico se incluye la pancreatectomía parcial o yeyunostomía pancreática ${ }^{8}$.

El objetivo de este estudio consiste en reportar

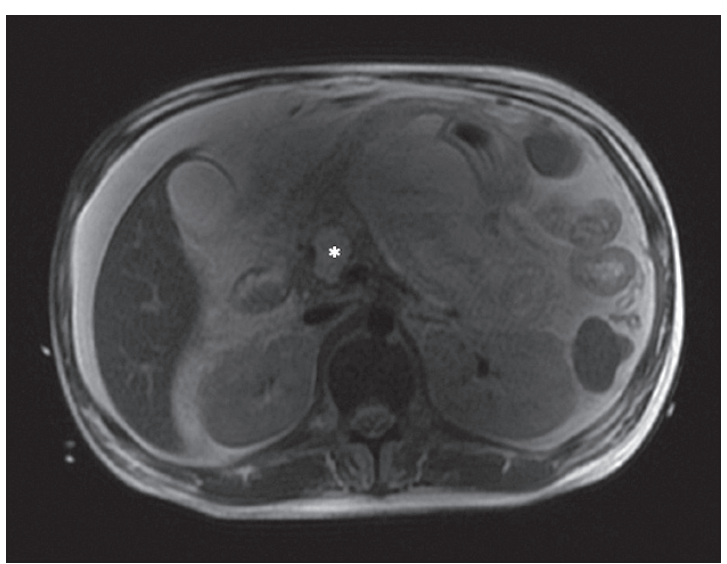

Figura 1. Corte axial en colangioresonancia magnética secuencia T2 que evidencia parénquima pancreático con disrupción del mismo a nivel del cuello del páncreas. La flecha verde indica colección quística dependiente del conducto de Wirsung. La cabeza de flecha indica el conducto de Wirsung dilatado. el caso de un hombre joven, con ascitis pancreática secundaria a lesión del conducto pancreático por trauma abdominal, tratado con éxito con terapia conservadora.

\section{Reporte de caso}

Paciente de sexo masculino de 21 años con antecedente de trauma abdominal cerrado un mes antes al ingreso, que consulta por distensión y dolor abdominal progresivo, hiporexia, emesis y pérdida de peso no cuantificada. Al examen físico, paciente hidratado, afebril, con abdomen distendido, onda ascítica positiva y sin signos de irritación peritoneal; paraclínicos con hiperamilasemia sérica de 3.740 $\mathrm{U} / \mathrm{L}$, por lo que se indica paracentesis con hallazgo de amilasa en líquido peritoneal de $23.316 \mathrm{U} / \mathrm{L}$.

Ante sospecha de lesión de conducto pancreático se solicitó pancreatografía por resonancia magnética que evidencia una alteración del segmento proximal del conducto pancreático principal asociado a lesión quística en el borde anterior de la unión de la cabeza con el cuerpo pancreático (Figura 1).

Se decidió iniciar manejo conservador y colocación de dren abdominal por radiología intervencionista para drenaje de líquido ascítico asociado a nutrición parenteral total y análogos de somatostatina

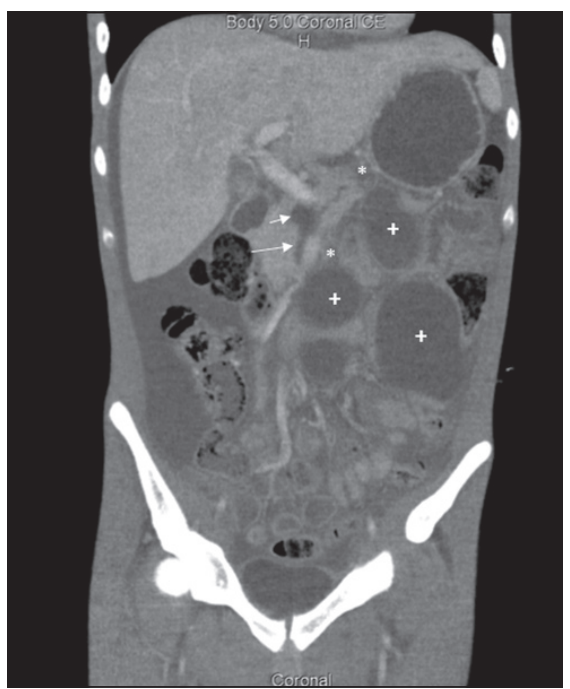

Figura 2. Tomografía abdominal corte coronal en fase portal Se observa dilatación del conducto de Wirsung (flecha larga) colección a nivel del proceso uncinado (flecha corta) con pérdida de la continuidad del parénquima pancreático a este nivel, edema de tejido graso peripancreático $\left({ }^{*}\right)$, múltiples colecciones líquidas infrapancreáticas $(+)$ y líquido libre en cavidad abdominal. 
(octreótido 100 ug subcutánea cada 8 h y somatostatina intravenosa en infusión continua a $210 \mathrm{ug} / \mathrm{h}$ ). A las tres semanas, paciente con evolución favorable y disminución de los drenajes por catéter. Se decide realizar tomografía abdominal multicorte en tres fases con protocolo para páncreas, encontrando colecciones por debajo del páncreas, con pérdida de la continuidad en el tercio proximal del parénquima pancreático y dilatación del conducto de Wirsung distal (Figura 2). Ante estos hallazgos se decide completar cuatro semanas de manejo conservador introduciendo y observando tolerancia completa de alimentación oral, con resolución completa de ascitis pancreática y niveles normales de enzimas pancreáticas en sangre.

\section{Discusión}

La Asociación Americana para la Cirugía del Trauma refiere que las lesiones del páncreas sin compromiso del conducto (grado I-II) se resuelven solo con manejo médico y las de grado III-IV-V generalmente requieren la colocación de un stent endoscópico o manejo quirúrgico ${ }^{7}$. La elección del manejo quirúrgico se basa en la integridad del conducto pancreático principal, la extensión del daño del parénquima pancreático, la ubicación anatómica de la lesión, la estabilidad del paciente y el grado de lesiones asociadas; la experiencia reportada del manejo endoscópico en la lesión del conducto pancreático es limitada, sin embargo, se ha evidenciado un uso exitoso del stent pancreático transpapilar en pacientes con fístula pancreática de hasta un $85 \% 0^{9,10}$.

El trauma pancreático se asocia con una alta morbimortalidad. Las muertes tempranas resultan de las lesiones vasculares intraabdominales y de otros órganos asociados y las muertes tardías por síndrome de respuesta inflamatoria sistémica secundaria a sepsis. Las complicaciones pancreáticas se pueden dividir en tempranas (pancreatitis, abscesos y fístulas) y tardías (pseudoquistes y rara vez deficiencia exocrina y endocrina $)^{11}$.

Bhasin y cols. ${ }^{3}$, propusieron un algoritmo de manejo para pacientes con trauma pancreático, en el que se evalúa inicialmente la integridad del conducto pancreático mediante colangiopancreatografía por resonancia magnética, aquellos sin lesión de este pueden ser tratados de manera conservadora, mientras que los pacientes con disrupciones parciales pueden beneficiarse de la colocación de un stent del conducto pancreático ${ }^{3,10}$, y en la interrupción completa del conducto se debe pensar en manejo quirúrgico definitivo ${ }^{3}$.
La ascitis pancreática se define como la acumulación de líquido pancreático en la cavidad peritoneal debido a la ruptura de un pseudoquiste o por el compromiso del conducto pancreático secundario al trauma. El diagnóstico requiere la demostración de líquido pancreático en la cavidad peritoneal que generalmente, se caracteriza por la elevación de la amilasa (mayor de $1.000 \mathrm{UI} / \mathrm{L}$ ) y de los niveles de proteína (mayor de $3 \mathrm{~g} / \mathrm{dL}$ ). El manejo de la ascitis pancreática puede ser inicialmente médico; sin embargo, con una tasa de fracaso reportada en la literatura del 40\%-60\% y una mortalidad hasta del $17 \%$, otro método terapéutico realizado es la terapia intervencionista como la colocación de un stent endoscópico en el conducto pancreático, la cual tiene una tasa de falla relativamente baja $(14,3 \%)$ y una tasa de mortalidad del $0 \%$; finalmente, el manejo puede ser quirúrgico con tasa de falla de $12,8 \%$ y una mortalidad reportada del $7,7 \%{ }^{12}$. Asimismo, la ascitis secundaria a trauma con lesión del conducto y fuga secundaria requiere cirugía en más del $50 \%$ de estos pacientes, con tasas de mortalidad del $15 \%$ al $25 \%{ }^{13}$. A pesar de que el manejo médico aislado tiene una tasa de éxito en la curación de la fístula pancreática que oscila entre el $17 \%$ y el $54 \%{ }^{7}$, en este caso se realizó este manejo en el tiempo límite propuesto por la literatura (2-3 semanas) con éxito y sin repercusión en la morbilidad del paciente.

\section{Conclusiones}

El manejo de las lesiones pancreáticas sigue siendo una encrucijada para la mayoría de los cirujanos y a pesar de los avances aún presenta altas tasas de morbilidad y mortalidad. Por lo tanto, la lesión del conducto pancreático principal debe sospecharse en todos los pacientes con trauma abdominal cerrado, incluso en aquellos que inicialmente se encuentren asintomáticos. El manejo debe realizarse con una planificación cuidadosa y exhaustiva antes de cualquier intervención, en lo posible deben ser atendidos por equipos multidisciplinarios con experiencia y recursos.

\section{Responsabilidades éticas}

Este estudio se apega a las normas éticas elaboradas en la declaración de Helsinki modificada (Brasil 2013) y al reporte de Belmont, asimismo, se ha preservado en todo momento anonimato y los autores han obtenido el consentimiento informado 
del paciente referido en el artículo, este documento obra en poder del autor de la correspondencia.

Conflictos de intereses: Los autores declaran no tener ningún conflicto de intereses.
Financiación: La presente investigación no ha recibido ayudas específicas provenientes de agencias del sector público, sector comercial o entidades sin ánimo de lucro.

\section{Bibliografía}

1. Larsen M, Kozarek R. Management of pancreatic ductal leaks and fistulae. J Gastroenterol Hepatol. 2014;29:1361-70. doi: 10.1111/jgh.12574.

2. Kim HS, Lee DK, Kim IW, et al. The role of endoscopic retrograde pancreatography in the treatment of traumatic pancreatic duct injury. Gastrointest Endosc. 2001;54(1):49-55. doi: 10.1067/ mge.2001.115733.

3. Bhasin DK, Rana SS, Rao C, et al. Endoscopic management of pancreatic injury due to abdominal trauma. JOP. 2012;13(2):187-92. PMID: 22406599.

4. Gougeon FW, Legros G, Archambault A, et al. Pancreatic trauma: a new diagnostic approach. Am J Surg. 1976;132(3):400-2. doi: 10.1016/0002-9610(76)90404-9.

5. Teh SH, Sheppard BC, Mullins RJ, et al. Diagnosis and management of blunt pancreatic ductal injury in the era of high- resolution computed axial tomography. Am J Surg. 2007;193(5):641-43. doi: 10.1016/j.amjsurg.2006.12.024.

6. Fulcher AS, Turner MA, Yelon

JA, et al. Magnetic resonance cholangiopancreatography (MRCP) in the assessment of pancreatic duct trauma and Its sequelae: preliminary findings. J Trauma. 2000;48(6):10017. doi: 10.1097/00005373-20000600000002.

7. Pata G, Casella C, Di Betta E, et al. Extension of nonoperative management of blunt pancreatic trauma to include grade III injuries : a safety analysis. World J Surg. 2009;33(8):1611-7. doi: 10.1007/ s00268-009-0082-7.

8. Telford JJ, Farrell JJ, Saltzman JR, et al. Pancreatic stent placement for duct disruption. Gastrointest Endosc. 2002;56(1):18-24. doi: 10.1067/ mge.2002.125107.

9. Testoni PA. Endoscopic Stenting in
Benign Pancreatic Diseases. JOP. 2007;8(1):141-50.

10. Bhasin DK, Rana SS, Rawal P. Endoscopic retrograde pancreatography in pancreatic trauma: Need to break the mental barrier. J Gastroenterol Hepatol. 2009;24(5):720-8. doi: 10.1111/j.14401746.2009.05809.x

11. Boffard KD, Brooks AJ. Pancreatic Trauma - Injuries to the Pancreas and Pancreatic Duct. Eur J Surg. 2000;166(1):4-12. doi: 10.1080/110241500750009627.

12. Karlapudi S, Hinohara T, Clements J, et al. Therapeutic challenges of pancreatic ascites and the role of endoscopic pancreatic stenting. BMJ Case Rep. 2014. Published online. doi: 10.1136/bcr-2014204774.

13. Kozarek RA, Jiranek GC, Traverso LW. Endoscopic treatment of pancreatic ascites. Am J Surg. 1994;168(3):223-6. doi: 10.1016/s0002-9610(05)80190-4. 